\title{
A Reverse Shock and Unusual Radio Properties in GRB 160625B
}

\author{
K. D. Alexander ${ }^{1}$ (1) , T. Laskar $^{2,3}$, E. Berger ${ }^{1}$ (1) , C. Guidorzi ${ }^{4}$, S. Dichiara ${ }^{5}$, W. Fong ${ }^{6,12}$ (1), A. Gomboc ${ }^{7}$, S. Kobayashi ${ }^{8}$ (1) \\ D. Kopac ${ }^{9}$, C. G. Mundell ${ }^{10}$, N. R. Tanvir ${ }^{11}$ (D), and P. K. G. Williams ${ }^{1}$ (D) \\ ${ }_{2}^{1}$ Harvard-Smithsonian Center for Astrophysics, 60 Garden Street, Cambridge, MA 02138, USA \\ ${ }^{2}$ National Radio Astronomy Observatory, 520 Edgemont Road, Charlottesville, VA 22903, USA \\ ${ }^{3}$ Department of Astronomy, University of California, 501 Campbell Hall, Berkeley, CA 94720-3411, USA \\ ${ }^{4}$ Dept. Physics and Earth Science, University of Ferrara, via Saragat 1, I-44122, Ferrara, Italy \\ ${ }^{5}$ Instituto de Astronomía, Universidad Nacional Autónoma de México, Ciudad de México, México \\ ${ }^{6}$ Steward Observatory, University of Arizona, 933 N. Cherry Avenue, Tucson, AZ 85721, USA \\ ${ }^{7}$ Centre for Astrophysics and Cosmology, University of Nova Gorica, Vipavska 11c, 5270 Ajdovščina, Slovenia \\ ${ }^{8}$ Astrophysics Research Institute, Liverpool John Moores University, IC2, Liverpool Science Park, 146 Brownlow Hill, Liverpool L3 5RF, UK \\ ${ }^{9}$ Faculty of Mathematics and Physics, University of Ljubljana, Jadranska 19, 1000 Ljubljana, Slovenia \\ ${ }^{10}$ Department of Physics, University of Bath, Claverton Down, Bath, BA2 7AY, UK \\ ${ }^{11}$ University of Leicester, Department of Physics \& Astronomy and Leicester Institute of Space \& Earth Observation, University Road, Leicester, LE1 7RH, UK \\ Received 2017 May 23; revised 2017 August 9; accepted 2017 August 31; published 2017 October 12
}

\begin{abstract}
We present multi-wavelength observations and modeling of the exceptionally bright long $\gamma$-ray burst GRB 160625B. The optical and X-ray data are well fit by synchrotron emission from a collimated blastwave with an opening angle of $\theta_{j} \approx 3.6$ and kinetic energy of $E_{K} \approx 2 \times 10^{51} \mathrm{erg}$, propagating into a low-density $\left(n \approx 5 \times 10^{-5} \mathrm{~cm}^{-3}\right)$ medium with a uniform profile. The forward shock is sub-dominant in the radio band; instead, the radio emission is dominated by two additional components. The first component is consistent with emission from a reverse shock, indicating an initial Lorentz factor of $\Gamma_{0} \gtrsim 100$ and an ejecta magnetization of $R_{B} \approx 1-100$. The second component exhibits peculiar spectral and temporal evolution and is most likely the result of scattering of the radio emission by the turbulent Milky Way interstellar medium (ISM). Such scattering is expected in any sufficiently compact extragalactic source and has been seen in GRBs before, but the large amplitude and long duration of the variability seen here are qualitatively more similar to extreme scattering events previously observed in quasars, rather than normal interstellar scintillation effects. High-cadence, broadband radio observations of future GRBs are needed to fully characterize such effects, which can sensitively probe the properties of the ISM and must be taken into account before variability intrinsic to the GRB can be interpreted correctly.
\end{abstract}

Key words: gamma-ray burst: general - gamma-ray burst: individual (GRB 160625B) - relativistic processes scattering

\section{Introduction}

Long-duration $\gamma$-ray bursts (GRBs) have been conclusively linked to the collapse of massive stars (Woosley \& Bloom 2006), but many questions about their progenitors and the physics powering GRB jets remain. The jet's composition and initial Lorentz factor can be probed directly through observations of synchrotron emission from the reverse shock (RS), produced when the jet begins to interact with the circumburst medium (Meszaros \& Rees 1993; Sari \& Piran 1999). Strong RS signatures are predicted when the energy density of the jet is dominated by baryons, while a weaker or absent RS may indicate a jet dominated by Poynting flux (Sari \& Piran 1999). $\mathrm{RS}$ emission fades quickly and later emission is dominated by the forward shock (FS) between the ejecta and the surrounding material (Sari et al. 1998; Sari \& Piran 1999), making early observations essential to constrain RS models.

The brightest RS signature is predicted in the optical band on $\lesssim$ hour timescales, but despite early optical observations enabled by robotic telescopes and rapid X-ray and ultraviolet (UV) localizations of GRBs by Swift, to date only a small fraction of GRBs exhibit unambiguous optical RS signatures (Japelj et al. 2014 and references therein). Bright optical flashes are now ruled out by observations in many events, while other

\footnotetext{
${ }^{12}$ Einstein Fellow.
}

events show complicated optical light curves that, like the prompt $\gamma$-ray emission, may originate instead from internal shocks (Kopač et al. 2013; Japelj et al. 2014). Some authors have proposed that RS emission may be easier to observe at longer wavelengths, where the emission peaks on timescales of days (Mundell et al. 2007; Melandri et al. 2010; Kopač et al. 2015). This approach was successfully adopted in trailblazing multi-frequency radio studies of GRB 130427A that characterized the RS emission at multiple epochs in detail (Laskar et al. 2013; Perley et al. 2014). In 2015, we began an intensive observing campaign at the Karl G. Jansky Very Large Array (VLA) to obtain additional early radio observations of long GRBs, resulting in a second multi-frequency detection of RS emission in GRB 160509A (Laskar et al. 2016).

Here, we present new results from our VLA campaign for the Fermi GRB 160625B. We combine our detailed multifrequency radio observations with optical and X-ray data, using a full MCMC statistical analysis to constrain the burst properties. The radio emission is dominated by a bright RS at early times and exhibits additional strong variability at late times, plausibly due to scattering by structures in the Galactic interstellar medium (ISM) along the line of sight. All error bars are $1 \sigma$ confidence intervals unless otherwise stated and all magnitudes are in the AB system (Oke \& Gunn 1983). We assume an event redshift of $z=1.406$ (determined from optical 
spectroscopy of the afterglow; Xu et al. 2016) and standard $\Lambda \mathrm{CDM}$ cosmology with $\Omega_{m}=0.27, \Omega_{\Lambda}=0.73$, and $H_{0}=$ $71 \mathrm{~km} \mathrm{~s}^{-1} \mathrm{Mpc}^{-1}$ throughout.

\section{GRB Properties and Observations}

\section{1. $\gamma$-Rays}

GRB 160625B was discovered by the Fermi Gamma-ray Space Telescope on 2016 June 25 (Dirirsa et al. 2016). The burst triggered the Gamma-ray Burst Monitor (GBM; Meegan et al. 2009) at 22:40:16.28 UTC and 22:51:16.03 UTC, and the Large Area Telescope (LAT; Atwood et al. 2009) at 22:43:24.82 UTC (Burns 2016). The burst was also detected by Konus-Wind, Integral, and CALET. The initial GBM trigger was a soft peak with a duration of $T_{90}=0.84 \mathrm{~s}$ and a fluence of $(1.75 \pm 0.05) \times 10^{-6} \mathrm{erg} \mathrm{cm}^{-2}(8 \mathrm{keV}-40 \mathrm{MeV})$. This precursor was followed by $\sim 180 \mathrm{~s}$ of quiescence and then by the main emission episode, which was extremely bright and had a duration of $T_{90}=35 \mathrm{~s}$ and a fluence of $(6.01 \pm 0.02) \times 10^{-4} \mathrm{erg} \mathrm{cm}^{-2}$. A third period of weak emission with a duration of $T_{90}=212 \mathrm{~s}$ and a fluence of $(5.65 \pm 0.02) \times 10^{-5} \mathrm{erg} \mathrm{cm}^{-2}$ followed after another $\sim 339 \mathrm{~s}$ gap (Zhang et al. 2016). For our analysis, we take $t_{0}$ to be the time of the LAT trigger, which coincides with the onset of the main emission episode, and take $T_{90}=35 \mathrm{~s}$ for the burst because this episode comprises $>90 \%$ of the high-energy emission. The total isotropic-equivalent energy of the prompt emission is $E_{\gamma, \text { iso }} \approx 3 \times 10^{54} \mathrm{erg}$ (Zhang et al. 2016). The prompt emission is discussed in detail in Zhang et al. (2016), Wang et al. (2017), and Lü et al. (2017).

\subsection{X-Ray: Swift/XRT}

The Swift X-Ray Telescope (XRT; Gehrels et al. 2004) began tiled observations of the Fermi error circle $2.5 \mathrm{hr}$ after the trigger and at $2.7 \mathrm{hr}$ detected a bright, uncataloged X-ray source determined to be the afterglow (Melandri et al. 2016). XRT continued to observe the afterglow for 47 days, with the last detection at 41.7 days. ${ }^{13}$ There are two breaks in the countrate light curve, at $t_{1} \approx 1.23 \times 10^{4} \mathrm{~s}$ and $t_{2} \approx 1.8 \times 10^{6} \mathrm{~s}$. The intervals $t<t_{1}$ and $t>t_{2}$ do not contain sufficient data to construct spectra with high enough signal-to-noise to rule out spectral evolution across the breaks, so we exclude these time ranges from our spectral analysis. We use the online tool from the Swift website (Evans et al. 2007, 2009) to extract a PC-mode spectrum from the time interval $t_{1}<t<t_{2}$ and fit the spectrum with a photoelectrically absorbed power-law model with the Galactic neutral hydrogen column fixed to $N_{\mathrm{H}, \mathrm{MW}}=9.76 \times 10^{20} \mathrm{~cm}^{-2}$ (Willingale et al. 2013). We determine the photon index to be $\Gamma_{\mathrm{X}}=1.86_{-0.09}^{+0.10}$ and the intrinsic absorption in the host galaxy to be $N_{\mathrm{H}, \text { int }}=2.1_{-1.8}^{+1.9} \times$ $10^{21} \mathrm{~cm}^{-2}$, with $90 \%$ confidence. $N_{\mathrm{H} \text {,int }}$ is consistent with zero at the $\sim 2 \sigma$ level, but we keep $N_{\mathrm{H} \text {,int }}=2.1 \times 10^{21} \mathrm{~cm}^{-2}$ when computing the counts-to-flux ratio. We use the corresponding spectral index $\beta_{\mathrm{X}}=1-\Gamma_{\mathrm{X}}=-0.86_{-0.10}^{+0.09}$ and the associated counts-to-absorbed flux ratio of $3.6 \times 10^{-11} \mathrm{erg} \mathrm{cm}^{-2} \mathrm{ct}^{-1}$ to convert the count rate to the observed flux density at $1 \mathrm{keV}$. The X-ray light curve is shown in Figure 1.

\footnotetext{
${ }^{13}$ http://www.swift.ac.uk/xrt_live_cat/00020667/
}
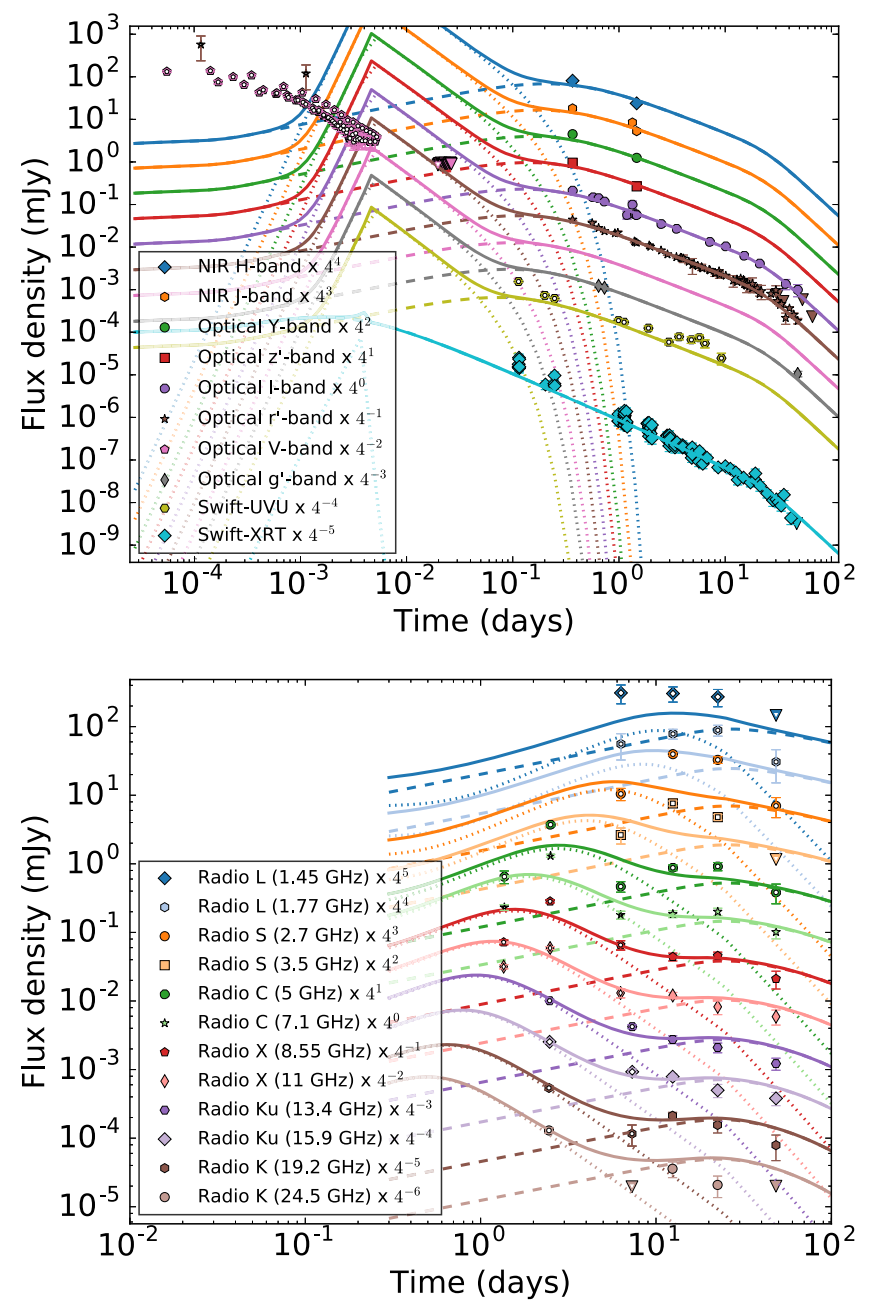

Figure 1. Light curves of GRB 160625B, vertically shifted for clarity. We take $t=0$ to be the LAT trigger time. The best-fit model (solid lines; Table 3) consists of a forward shock (dashed component) and a Newtonian reverse shock (dotted component; Model 1). The optical and X-ray data drive the properties of the forward shock (top), while the reverse shock dominates the radio emission at early times (bottom). The optical detections before 0.01 day are likely related to the prompt emission, consistent with the sub-dominant extrapolated flux of the reverse shock at early times. These early data are excluded from our model fitting, as is the portion of the radio emission showing evidence of multiple components. The excluded points are indicated with open symbols.

\subsection{UV/Optical: Swift/UVOT}

The Swift UV/Optical Telescope (UVOT; Roming et al. 2005) began observing the burst $2.7 \mathrm{hr}$ after the Fermi trigger, detecting a bright source in $U$ band (Oates 2016). Additional observations were conducted in the $U, W 1, M 2$, and $V$ filters. The photometry was complicated by the presence of a nearby bright star, which created reflections that dominated the counts at the source position in many images and rendered the bluer bands entirely unusable. We restrict our analysis to the $U$ band images, where the source is clearly detected and the background is more uniform.

We analyze the $U$ band data using HEASoft (v. 6.16). We perform photometry with a $5^{\prime \prime}$ aperture and a $15^{\prime \prime}$ background region. We vary the position of the background region from image to image to avoid reflection artifacts from the nearby bright star and most closely match the background near the GRB, but caution that the flux errors thus obtained may be 
underestimated. Given the large systematic uncertainties, we do not include these data in our model fitting, but they are shown for completeness in Figure 1.

\subsection{Optical/NIR: LCOGT, ORM, Magellan, GCN Circulars}

We began observing GRB 160625B with the $2 \mathrm{~m}$ Faulkes Telescope North (FTN), which is operated by Las Cumbres Observatory Global Network (LCOGT; Brown et al. 2013) on June 26.01 UT (0.56 days after the GRB) in the SDSS $r^{\prime}$ and $i^{\prime}$ filters. Observations with the FTN went on on a daily basis for almost a week, then the $2 \mathrm{~m}$ Liverpool Telescope (LT; Steele et al. 2004) at the Observatorio del Roque de Los Muchachos (ORM) took over in the same filters with a cadence of a few days until 37 days post GRB. Bias and flat-field corrections were applied using the specific pipelines of the LCOGT and of the LT. The optical afterglow magnitudes were obtained by PSF-fitting photometry, after calibrating the zero-points with nine nearby stars with SDSS $r^{\prime}$ and $i^{\prime}$ magnitudes from the URAT1 catalog (Zacharias et al. 2015). A systematic error of $0.02 \mathrm{mag}$, due to the zero-point scatter of the calibrating stars, was added to the statistical uncertainties of magnitudes.

We subsequently observed GRB 160625B on 2016 August 12.12 UT (48.1 day after the burst) with LDSS3 on the $6.5 \mathrm{~m}$ Magellan/Clay Telescope at Las Campanas Observatory. We obtained eight $180 \mathrm{~s}$ exposures in $i^{\prime}$ band, six $240 \mathrm{~s}$ exposures in $r^{\prime}$ band, and four $420 \mathrm{~s}$ exposures in $g^{\prime}$ band. The data were reduced using a custom IDL script and standard IRAF routines. The afterglow is detected in a stacked image in each filter. Aperture photometry was performed using nearby stars from the Pan-STARRS $3 \pi$ survey (Chambers et al. 2016).

Finally, we collected other optical and near-infrared (NIR) observations of GRB 160625B reported through the Gammaray Burst Coordinates Network (GCN) Circulars and by Zhang et al. (2016) and converted all photometry to flux densities. These observations include early optical data from the Pi of the Sky North observatory (Batsch et al. 2016) and the MiniMegaTORTORA telescope (Karpov et al. 2016; Zhang et al. 2016), which detected a bright optical flash coincident with the main peak of $\gamma$-ray emission. The flux densities derived from the Mini-MegaTORTORA photometry are systematically $\sim 1.5$ times larger than flux densities from the simultaneous Pi of the Sky observations; this offset is due to either a calibration difference or the different filter bandpasses used by each instrument. Both groups used reference stars to perform a color correction and obtain approximate $V$ band magnitudes, but without a simultaneous spectrum an absolute photometric calibration is not possible. A precise calibration is not necessary for our results, as we only include these data in our modeling as an approximate upper limit on RS emission (Section 5.1). We list our Las Cumbres, ORM, and Magellan observations in Table 1. The fluxes reported in Table 1 have not been corrected for extinction, as this correction is included directly in our modeling framework (Section 4). We expect moderate Galactic extinction along the line of sight to the GRB: $A_{g}=0.42, A_{r}=0.29, A_{i}=0.22$, and $A_{z}=0.16$ (Schlafly \& Finkbeiner 2011). The optical light curves including all of the data used in our modeling are shown in Figure 1.

\subsection{Radio: VLA}

We observed the afterglow using the Karl G. Jansky VLA starting 1.35 days after the burst. Our observations span frequencies between 1.45 and $24.5 \mathrm{GHz}$ and extend to 48.38 days after the burst. The data were analyzed with the Common Astronomy Software Applications (CASA) using 3C48 or 3C286 as a flux calibrator (depending on the LST start time of each observation) and $\mathrm{J} 1810+5649$ as a gain calibrator. The flux densities and associated uncertainties were determined using the imtool program within the pwkit package ${ }^{14}$ (version 0.8.4.99; Williams et al. 2017) and are reported in Table 2. The radio light curves are shown in Figure 1 and the radio spectral energy distributions (SEDs) at the various epochs are shown in Figure 2.

\section{Basic Considerations}

We interpret our multi-wavelength observations using a standard synchrotron emission model (Sari et al. 1998; Granot \& Sari 2002). In this model, the emitting electrons are assumed to have been accelerated into a non-thermal distribution $N(\gamma) \propto \gamma^{-p}$ for $\gamma>\gamma_{m}$, where $\gamma_{m}$ is the minimum Lorentz factor of the distribution. The resulting SED is described by three break frequencies (the self-absorption frequency, $\nu_{\mathrm{a}}$, the characteristic synchrotron frequency, $\nu_{m}$, and the cooling frequency, $\nu_{\mathrm{c}}$ ) and an overall flux normalization. The temporal evolution of these quantities depends on the circumburst density profile and the outflow geometry. In this section, we estimate basic properties of the afterglow and consider two possible models for the circumburst medium: a constant density ISM profile (Sari et al. 1998) and a wind profile where the density scales as $r^{-2}$ (Chevalier \& Li 2000).

\subsection{Time of Jet Break}

The X-ray, $r^{\prime}$, and $i^{\prime}$ band light curves all steepen at $t \approx 25$ day, suggestive of a jet break. The best constraints on the break timing and post-break decline rate come from the $r^{\prime}$ band light curve, which can be fit by two power-law segments with a break at $t_{\text {jet }}=27 \pm 2$ day. Before the break, the decline rate is $\alpha_{1, \mathrm{r}}=-0.94 \pm 0.01$; after the break, it steepens to $\alpha_{2, \mathrm{r}}=-2.3 \pm 0.4\left(\Delta \alpha_{12, \mathrm{r}}=-1.4 \pm 0.4\right)$. The steep postbreak decline rate and the lack of flattening at late times indicate that the GRB host contributes negligibly to the total flux. By $t=t_{\text {jet }}$, we expect $\nu_{\mathrm{m}}$ to be located below the optical band, and the $r^{\prime}$ band light curve should therefore evolve as $t^{-p}$ after the jet break (Sari et al. 1999). We therefore estimate $p \approx 2.3$ for the non-thermal electron distribution.

The radio observations also show evidence of a jet break, as the flux declines at all frequencies between 22 and 48 days. The higher frequencies $(\nu>7 \mathrm{GHz})$ prefer a significantly earlier jet break time than the optical and X-ray observations, $t_{\text {jet }} \approx 12$ days; other effects dominate the emission at frequencies below $7 \mathrm{GHz}$ during this time range (see Section 5.) Such an earlier jet break would require the presence of an additional component to explain the smooth decline of the optical and X-ray emission at $t \approx 12-27$ day. However, this explanation is disfavored due to its increased complexity and as there are other signs of unusual variability in the radio, we take $t_{\text {jet }} \approx 25$ days as preferred by the optical and X-ray data.

\footnotetext{
${ }^{14}$ Available at https://github.com/pkgw/pwkit.
} 
Table 1

Optical Observations

\begin{tabular}{|c|c|c|c|c|c|c|}
\hline $\begin{array}{l}t \\
\text { (day) }\end{array}$ & Observatory & Instrument & Filter & $\begin{array}{l}\text { Magnitude } \\
\text { (AB) }\end{array}$ & $\begin{array}{c}\text { Frequency } \\
\left(10^{14} \mathrm{~Hz}\right)\end{array}$ & $\begin{array}{l}\text { Flux Density } \\
(\mu \mathrm{Jy})\end{array}$ \\
\hline 0.56 & LCOGT & FTN & $r^{\prime}$ & $18.49 \pm 0.12$ & 4.56 & $146 \pm 17$ \\
\hline 0.57 & LCOGT & FTN & $i^{\prime}$ & $18.47 \pm 0.14$ & 3.93 & $150 \pm 20$ \\
\hline 1.19 & LCOGT & FTN & $i^{\prime}$ & $19.56 \pm 0.04$ & 3.93 & $57 \pm 12$ \\
\hline 1.40 & LCOGT & FTN & $r^{\prime}$ & $19.51 \pm 0.20$ & 4.56 & $60 \pm 3$ \\
\hline 1.41 & LCOGT & FTN & $i^{\prime}$ & $19.46 \pm 0.05$ & 3.93 & $60 \pm 3$ \\
\hline 1.42 & LCOGT & FTN & $r^{\prime}$ & $19.60 \pm 0.04$ & 4.56 & $53 \pm 3$ \\
\hline 1.46 & LCOGT & FTN & $i^{\prime}$ & $19.48 \pm 0.03$ & 3.93 & $59 \pm 3$ \\
\hline 2.49 & LCOGT & FTN & $r^{\prime}$ & $20.09 \pm 0.06$ & 4.56 & $33.4 \pm 1.9$ \\
\hline 2.50 & LCOGT & FTN & $i^{\prime}$ & $20.04 \pm 0.10$ & 3.93 & $35 \pm 3$ \\
\hline 3.47 & LCOGT & FTN & $r^{\prime}$ & $20.48 \pm 0.04$ & 4.56 & $23.3 \pm 1.2$ \\
\hline 3.49 & LCOGT & FTN & $i^{\prime}$ & $20.32 \pm 0.09$ & 3.93 & $27 \pm 2$ \\
\hline 4.54 & LCOGT & FTN & $r^{\prime}$ & $20.75 \pm 0.11$ & 4.56 & $18.2 \pm 1.9$ \\
\hline 5.52 & LCOGT & FTN & $r^{\prime}$ & $21.00 \pm 0.13$ & 4.56 & $14.5 \pm 1.8$ \\
\hline 7.17 & ORM & LT & $i^{\prime}$ & $21.09 \pm 0.03$ & 3.93 & $13.3 \pm 0.7$ \\
\hline 7.18 & ORM & LT & $r^{\prime}$ & $21.26 \pm 0.03$ & 4.56 & $11.4 \pm 0.6$ \\
\hline 10.12 & ORM & $\mathrm{LT}$ & $i^{\prime}$ & $21.35 \pm 0.03$ & 3.93 & $10.5 \pm 0.5$ \\
\hline 10.13 & ORM & LT & $r^{\prime}$ & $21.57 \pm 0.03$ & 4.56 & $8.6 \pm 0.4$ \\
\hline 15.13 & ORM & LT & $i^{\prime}$ & $21.9 \pm 0.08$ & 3.93 & $6.3 \pm 0.5$ \\
\hline 15.14 & ORM & LT & $r^{\prime}$ & $22.06 \pm 0.05$ & 4.56 & $5.5 \pm 0.3$ \\
\hline 21.09 & ORM & LT & $i^{\prime}$ & $22.36 \pm 0.10$ & 3.93 & $4.1 \pm 0.4$ \\
\hline 21.10 & ORM & LT & $r^{\prime}$ & $22.64 \pm 0.12$ & 4.56 & $3.2 \pm 0.4$ \\
\hline 37.10 & ORM & LT & $i^{\prime}$ & $23.56 \pm 0.26$ & 3.93 & $1.4 \pm 0.4$ \\
\hline 37.12 & ORM & $\mathrm{LT}$ & $r^{\prime}$ & $24.05 \pm 0.28$ & 4.56 & $0.9 \pm 0.3$ \\
\hline 48.13 & Magellan & LDSS3 & $i^{\prime}$ & $23.9 \pm 0.3$ & 3.93 & $1.0 \pm 0.3$ \\
\hline 48.15 & Magellan & LDSS3 & $r^{\prime}$ & $24.23 \pm 0.15$ & 4.56 & $0.74 \pm 0.11$ \\
\hline 48.18 & Magellan & LDSS3 & $g^{\prime}$ & $24.33 \pm 0.15$ & 6.29 & $0.67 \pm 0.10$ \\
\hline
\end{tabular}

Note. Optical observations of GRB 160625B from Las Cumbres Observatory (LCOGT), the Observatorio del Roque de Los Muchachos (ORM), and Magellan. All values of $t$ are relative to 2016 June 25 22:43:24.82 UT, the LAT trigger time. The data have not been corrected for extinction.

\subsection{Circumburst Density Profile, Location of $\nu_{\mathrm{c}}$, and Host Extinction}

Prior to $t=t_{\text {jet }}$, the optical and X-ray light curves can each be fit with a single power law. The $i^{\prime}$ band light curve has a similar decline rates to the $r^{\prime}$ band light curve, $\alpha_{1, i}=-0.94 \pm 0.02$, while the X-ray light curve declines more steeply, with $\alpha_{1, \mathrm{XRT}}=-1.24 \pm 0.02$. A natural explanation for this in the context of the synchrotron model is that the cooling break $\left(\nu_{\mathrm{c}}\right)$ is located between the optical and X-ray bands. The predicted decline rate for $\nu<\nu_{\mathrm{c}}$ depends on the circumburst density profile and is $\alpha_{\mathrm{ISM}}=3(1-p) / 4$ for an ISM profile and $\alpha_{\text {wind }}=(1-3 p) / 4$ for a wind profile (Granot \& Sari 2002). Using the $r^{\prime}$ band light curve, we find $p=2.25 \pm 0.02$ for the ISM case and $p=1.59 \pm 0.02$ for the wind case. For both profiles, the predicted decline rate for $\nu>\nu_{\mathrm{c}}$ is $\alpha=(2-3 p) / 4$ and the X-ray decline rate implies $p=2.32 \pm 0.03$. The pre-jet break optical and X-ray observations are thus only self-consistent if the circumburst medium is ISM-like rather than wind-like, giving $p \approx 2.3$ in agreement with the value derived from the post-jet break decline rate in Section 3.1. We therefore only consider the ISM profile for our detailed modeling in Sections 4 and 5 .

We can also use the inferred value of $p$ and the optical/NIR SED to constrain the amount of extinction in the GRB host. For $\nu<\nu_{c}$ and zero extinction, the predicted spectral index is $\beta=-0.65$ for $p=2.3$. Fitting the RATIR rizYJH data points at 1.468 day (Watson et al. 2016), we find a spectral index of $\beta_{\mathrm{NIR}}=-0.68 \pm 0.07$, consistent with this value. We see a slightly steeper $r-g$ spectral index in MITSuME observations at 0.731 day (Kuroda et al. 2016), $\beta_{r g}=-1.0 \pm 0.2$. This indicates a small total amount of extinction along the line of sight, consistent with the expected amount of Galactic extinction (Section 2.4) and little to no extinction in the GRB host galaxy. The spectral index in the XRT $0.3-10 \mathrm{keV}$ band is $\beta_{\mathrm{X}}=-0.86_{-0.10}^{+0.09}$, which is intermediate between the values expected for $p \approx 2.3$ when $\nu_{\mathrm{X}}<\nu_{\mathrm{c}}\left(\beta_{\mathrm{X}} \approx-0.65\right)$ and $\nu_{\mathrm{X}}>\nu_{\mathrm{c}}\left(\beta_{\mathrm{X}} \approx-1.15\right)$. This may indicate that $\nu_{\mathrm{c}}$ is located only slightly below the $\mathrm{X}$-ray band, as the spectrum is expected to transition smoothly from one power-law index to the other around each break frequency. The NIR to X-ray spectral index is $\beta_{\text {NIR }-\mathrm{X}}=-0.71 \pm 0.01$, slightly steeper than expected if $\nu_{\mathrm{X}}<\nu_{\mathrm{c}}$ for $p \approx 2.3$. Therefore $\beta_{\mathrm{NIR}-\mathrm{X}}$ is also consistent with $\nu_{c}$ being located just below the X-ray band.

\subsection{Multiple Radio Components}

The radio emission at $t=2.48$ day is dominated by a single component with a spectral peak around $6 \mathrm{GHz}$. If the emission is fit with a broken power law and the spectral index above the peak is extrapolated to high frequencies, this component underpredicts the observed optical and X-ray emission by several orders of magnitude (Figure 3, top). We therefore conclude that a separate mechanism is required to explain the radio emission at $t \leqslant 7$ day and show in Section 5.1 that this component is consistent with a RS. The peak of this component must be above $11 \mathrm{GHz}$ at 1.36 day, implying that the peak frequency evolves faster than $t^{-1}$. This means that $\nu_{p} \lesssim 2 \mathrm{GHz}$ at 6.8 day and $\nu_{p} \lesssim 1 \mathrm{GHz}$ at 12.46 day, indicating that this component cannot contribute significantly to the observed radio emission after 7 days. 
Table 2

Radio Observations

\begin{tabular}{|c|c|c|}
\hline $\begin{array}{l}t \\
\text { (day) }\end{array}$ & $\begin{array}{c}\text { Frequency } \\
\quad(\mathrm{GHz})\end{array}$ & $\begin{array}{c}\text { Flux Density } \\
(\mu \mathrm{Jy})\end{array}$ \\
\hline 1.37 & 5.0 & $163 \pm 34$ \\
\hline 1.37 & 7.1 & $232 \pm 22$ \\
\hline 1.35 & 8.5 & $288 \pm 23$ \\
\hline 1.35 & 11.0 & $507 \pm 35$ \\
\hline 2.50 & 5.0 & $932 \pm 24$ \\
\hline 2.50 & 7.1 & $1310 \pm 20$ \\
\hline 2.49 & 8.5 & $1135 \pm 28$ \\
\hline 2.49 & 11.0 & $946 \pm 25$ \\
\hline 2.47 & 13.5 & $646 \pm 25$ \\
\hline 2.47 & 16.0 & $650 \pm 19$ \\
\hline 2.45 & 19.2 & $553 \pm 34$ \\
\hline 2.45 & 24.5 & $530 \pm 47$ \\
\hline 6.31 & 1.45 & $300 \pm 90$ \\
\hline 6.31 & 1.77 & $200 \pm 90$ \\
\hline 6.30 & 2.68 & $164 \pm 33$ \\
\hline 6.30 & 3.52 & $165 \pm 43$ \\
\hline 6.29 & 5.0 & $117 \pm 21$ \\
\hline 6.29 & 7.1 & $180 \pm 24$ \\
\hline 6.28 & 8.5 & $262 \pm 41$ \\
\hline 6.28 & 11.0 & $209 \pm 32$ \\
\hline 7.32 & 13.5 & $270 \pm 18$ \\
\hline 7.32 & 16.0 & $237 \pm 23$ \\
\hline 7.30 & 19.2 & $119 \pm 40$ \\
\hline 7.30 & 24.5 & $80 \pm 27$ \\
\hline 12.50 & 1.45 & $297 \pm 74$ \\
\hline 12.50 & 1.77 & $307 \pm 50$ \\
\hline 12.49 & 2.68 & $621 \pm 31$ \\
\hline 12.49 & 3.52 & $475 \pm 40$ \\
\hline 12.48 & 5.0 & $219 \pm 21$ \\
\hline 12.48 & 7.1 & $185 \pm 21$ \\
\hline 12.47 & 8.5 & $176 \pm 23$ \\
\hline 12.47 & 11.0 & $193 \pm 21$ \\
\hline 12.45 & 13.5 & $176 \pm 23$ \\
\hline 12.45 & 16.0 & $202 \pm 21$ \\
\hline 12.43 & 19.2 & $218 \pm 26$ \\
\hline 12.43 & 24.5 & $147 \pm 38$ \\
\hline 22.52 & 1.45 & $265 \pm 75$ \\
\hline 22.52 & 1.77 & $346 \pm 62$ \\
\hline 22.51 & 2.68 & $512 \pm 57$ \\
\hline 22.51 & 3.52 & $300 \pm 27$ \\
\hline 22.50 & 5.0 & $229 \pm 31$ \\
\hline 22.50 & 7.1 & $201 \pm 25$ \\
\hline 22.49 & 8.5 & $183 \pm 24$ \\
\hline 22.49 & 11.0 & $132 \pm 30$ \\
\hline 22.47 & 13.5 & $134 \pm 22$ \\
\hline 22.47 & 16.0 & $128 \pm 28$ \\
\hline 22.45 & 19.2 & $159 \pm 38$ \\
\hline 22.45 & 24.5 & $85 \pm 30$ \\
\hline 48.38 & 1.45 & $142 \pm 47$ \\
\hline 48.38 & 1.77 & $120 \pm 61$ \\
\hline 48.37 & 2.68 & $109 \pm 35$ \\
\hline 48.37 & 3.52 & $72 \pm 24$ \\
\hline 48.36 & 5.0 & $96 \pm 31$ \\
\hline 48.36 & 7.1 & $101 \pm 21$ \\
\hline 48.35 & 8.5 & $84 \pm 25$ \\
\hline 48.35 & 11.0 & $95 \pm 23$ \\
\hline 48.33 & 13.5 & $78 \pm 16$ \\
\hline 48.33 & 16.0 & $97 \pm 21$ \\
\hline 48.31 & 19.2 & $81 \pm 33$ \\
\hline 48.31 & 24.5 & $82 \pm 27$ \\
\hline
\end{tabular}

Note. VLA observations of GRB 160625B. All values of $t$ are relative to the LAT trigger time, 2016 June 25 22:43:24.82 UT.

We also observe a low-frequency rebrightening at 12-22 day peaked at $\sim 3 \mathrm{GHz}$, which appears distinct from higher-frequency emission at that time (Figure 3, bottom). The high-frequency emission is broadly consistent with expectations for the FS. The low-frequency emission cannot be the same component dominating the radio emission before 7 days unless that component's peak frequency were to start increasing in time after 7 days; such behavior is not predicted for either FS or RS emission and would be unprecedented in GRB afterglow studies. This component is also too spectrally narrow for standard synchrotron emission: for the broken power-law fit in Figure 3, we find that the spectral index is $\beta_{1}=3.0 \pm 0.1$ below the peak and $\beta_{2}=-3.7 \pm 0.6$ above it. Together, these properties suggest distortion of the intrinsic low-frequency radio SED by interstellar scintillation (ISS) as the emission propagates through the turbulent Galactic ISM (see the review by Rickett 1990). ISS is known to cause strong, uncorrelated flux density variations in GRB afterglows and other sufficiently compact radio sources and should be carefully considered before claiming that observed rapid spectral and temporal variations require exotic new effects intrinsic to the GRB. We discuss ISS and other possible origins of this component in more detail in Section 5.2.

\section{FS Model}

Motivated by these basic considerations, we model the afterglow as synchrotron emission resulting from the FS between the jet ejecta and the surrounding medium, including the effects of inverse Compton cooling (Sari \& Esin 2001; Granot \& Sari 2002). Our modeling framework is described in detail in Laskar et al. (2014) and Laskar et al. (2015) and uses the Python package emcee (Foreman-Mackey et al. 2013) to fully explore parameter space and uncover correlations between physical parameters. The model parameters are the isotropicequivalent ejecta kinetic energy $\left(E_{\mathrm{K}, \text { iso }}\right)$, the circumburst density $\left(n_{0}\right)$, the electron energy index $(p)$, the jet break time $\left(t_{\text {jet }}\right)$, and the fraction of the shock energy imparted to electrons $\left(\epsilon_{e}\right)$ and magnetic fields $\left(\epsilon_{B}\right)$. We include a correction for Galactic extinction but fix the extinction in the GRB host to $A_{V}=0$, as the data strongly prefer negligible host extinction if this parameter is allowed to vary freely (consistent with Section 3.2). We also require $\epsilon_{e}<\frac{1}{3}$ and $\epsilon_{B}<\frac{1}{3}$, their equipartition values. This is commonly done to partially break parameter degeneracies that arise when one or more of the FS break frequencies is not well constrained (e.g., Laskar et al. 2015) and is consistent with recent work that finds most GRBs have $\epsilon_{e}=0.13-0.15$ (Beniamini \& van der Horst 2017). We exclude the radio data at early times $(t<12$ days $)$ and all data at frequencies below $7 \mathrm{GHz}$ because other components dominate this emission (Section 5). We also exclude the $U$ band data due to the systematic uncertainties discussed in Section 2.3. The parameters for our best-fit model $\left(\chi^{2}=7.56\right.$ for 6 degrees of freedom) are listed in Table 3 and the model light curves are shown in Figure 1. All data points excluded from our model fitting are marked with open symbols in Figure 1. The full marginalized posterior probability density functions for each model parameter and two additional derived parameters (the jet opening angle, $\theta_{\text {jet }}$, and the beamingcorrected kinetic energy, $E_{K}$ ) are given in Figure 4. Correlations between the physical parameters $E_{\mathrm{K} \text {,iso }}, n_{0}, \epsilon_{e}$, and $\epsilon_{B}$ are shown in Figure 5.

The self-absorption frequency $\nu_{a}$ is located below the radio band for the entirety of our observations and is therefore poorly constrained. This creates degeneracies between $\epsilon_{e}, \epsilon_{B}, n_{0}$, and $E_{\mathrm{K} \text {,iso, }}$ as illustrated in Figure 5. This also leads to a large 

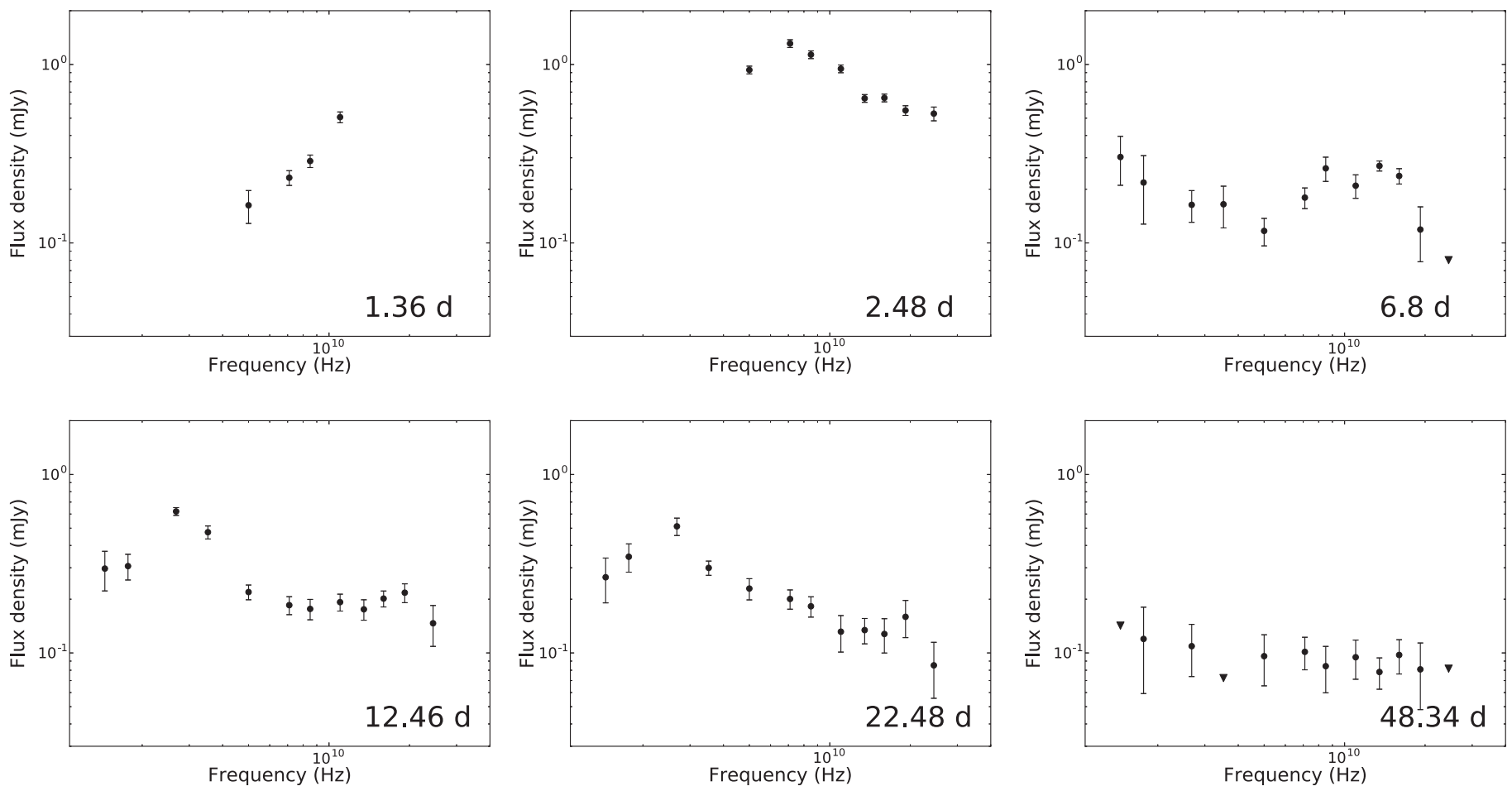

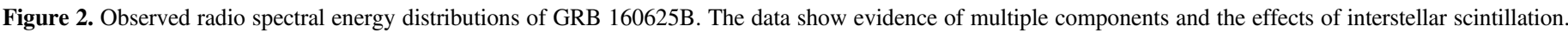

uncertainty in the strength of inverse Compton cooling, with possible Compton $Y$ parameter values ranging from $Y \approx 0.2$ (mildly significant cooling) to $Y \approx 20$ (strong cooling). Our best-fit model has $Y \approx 3.7$, which is comparable to the value recently found for GRB $160509 \mathrm{~A}(Y \approx 2.4)$ and corresponds to moderately significant cooling (Laskar et al. 2016). We find $p=2.31 \pm 0.01$ and $t_{\text {jet }}=25 \pm 1$ days, in agreement with the arguments presented in Section 3. The kinetic energy of the outflow is $E_{\mathrm{K}, \text { iso }}=\left(1.1_{-0.5}^{+1.0}\right) \times 10^{54} \mathrm{erg}$, similar to the energy released in the prompt emission of this GRB, $E_{\gamma, \text { iso }} \approx 3 \times 10^{54} \mathrm{erg}$ (Zhang et al. 2016). This implies a high radiative efficiency for the burst of $\eta_{\gamma}=E_{\gamma, \text { iso }} /\left(E_{\mathrm{K}, \text { iso }}+\right.$ $\left.E_{\gamma, \text { iso }}\right)=0.73_{-0.14}^{+0.10}$, which is within the range of efficiencies found for long GRBs in previous work (Zhang et al. 2007). The beaming-corrected outflow kinetic energy is $\left(2.3_{-1.2}^{+1.8}\right) \times$ $10^{51} \mathrm{erg}$. The density implied by the model is quite low, $n_{0}=(5 \pm 3) \times 10^{-5} \mathrm{~cm}^{-3}$. Previous studies have found that the circumburst density varies widely among long GRBs, with estimates for individual bursts ranging from $10^{-5}$ to $10^{3} \mathrm{~cm}^{-3}$ (Laskar et al. 2014, 2015). GRB 130427A and GRB 160509A, which both had strong detections of RS emission in the radio, had very low densities of $\approx 10^{-3} \mathrm{~cm}^{-3}$, suggesting that lowdensity environments may be required to produce observable, long-lasting RS emission (Laskar et al. 2013, 2016). As we will see in Section 5.1, GRB 160625B likely also has a strong RS.

\section{Multiple Radio Components}

The early radio observations $(t<12$ days) at all frequencies and the low-frequency radio observations $(\nu<7 \mathrm{GHz})$ at all times are not well fit by the FS model discussed in Section 4. A natural explanation for the radio excess at early times is emission from a RS. As a RS alone cannot explain all of the data, we also consider how propagation through the ISM of the Galaxy affects the radio emission via scintillation.

\subsection{Early Radio Emission: A RS}

We first model the excess radio emission in the early epochs as synchrotron emission from a RS. The RS is launched when the GRB ejecta first begin to interact with the surrounding medium and propagates through the ejecta, probing the properties of the jet itself (Sari \& Piran 1999; Kobayashi \& Sari 2000). In GRB 160625B, the RS model is constrained by both radio observations and early optical observations. The onset of the optical emission is closely tied to the onset of the main episode of prompt $\gamma$-ray emission; observations by the Mini-MegaTORTORA telescope reveal that the optical flux density increased by a factor of $>90$ in the $30 \mathrm{~s}$ prior to the LAT trigger and peaked $\approx 12 \mathrm{~s}$ after the LAT trigger time $(\approx 3 \mathrm{~s}$ after the $\gamma$-ray peak; Zhang et al. 2016). This is inconsistent with RS emission models because $T_{90}=35 \mathrm{~s}$ and the RS optical emission is expected to peak at $t_{\mathrm{dec}} \geqslant T_{90}$ (Sari \& Piran 1999). We therefore conclude (as do Lü et al. 2017) that the early optical flash is related to the prompt emission and treat it as an upper bound to the RS emission.

The RS is most clearly detected in the radio in epochs 1 and 2 , so we begin our analysis by fitting this component in these two epochs and then propagate the RS backwards and forwards in time. The radio observations at 1.4 days can be fit with a steeply rising power law with a spectral index $\beta \approx 2$, implying that $\nu_{a, \mathrm{RS}} \gtrsim 11 \mathrm{GHz}$ at this time. Fitting the epoch 2 radio SED with a broken power law, we find that the SED peaks at $\approx 6 \mathrm{GHz}$ and the spectral index above the peak frequency is $\beta \approx-0.9$. This implies that the peak at 2.5 days is most likely $\nu_{a}$ (Model 1). In this case, the SED shape also requires $\nu_{m} \lesssim 6 \mathrm{GHz}$ and $\nu_{c} \gtrsim 25 \mathrm{GHz}$ at 2.5 days. A second possibility is that the peak is $\nu_{c}$ (Model 2). In this case, $\nu_{a} \gtrsim 6 \mathrm{GHz}$ at 2.5 days and $\nu_{m}$ is unconstrained because the spectrum cuts off above $\nu_{c}$. This means that various RS models can fit the data equally well, but we show that some models can be ruled out by physical considerations. 

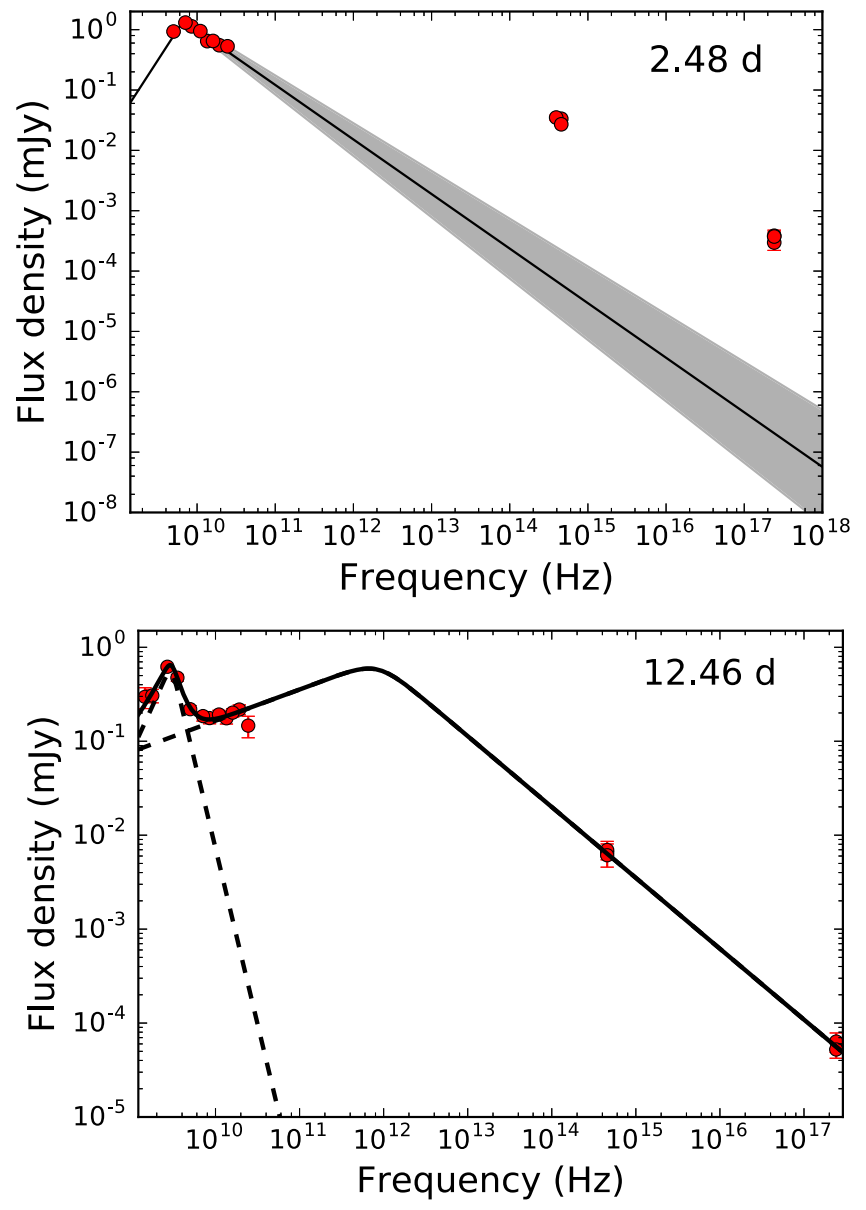

Figure 3. Top: the radio to X-ray spectral energy distribution at 2.48 day. The radio data are fit with a broken power law and the spectral index above $6 \mathrm{GHz}$ is extended to the optical and X-ray bands (black line, shaded region indicates the $1 \sigma$ uncertainty in the fit). The fit underpredicts the optical and X-ray emission by several orders of magnitude, indicating that the radio emission is dominated by a separate component at this time. Bottom: the radio to X-ray spectral energy distribution at 12.46 day fit with two components. The radio data above $8 \mathrm{GHz}$ connect simply to the optical and X-ray data with a $\nu^{1 / 3}$ power law transitioning to a $\nu^{-0.75}$ power law, as expected for the forward shock. The radio data below $8 \mathrm{GHz}$ require a second, extremely spectrally narrow component that does not connect simply to the FS or to the component dominating the radio emission at 2.48 days.

In both cases, we run into problems when we attempt to connect the observed SEDs at different epochs. The temporal evolution of the emission depends on whether the RS is relativistic in the frame of the unshocked ejecta. The evolution of the shocked ejecta in a Newtonian RS is characterized by the parameter $g$, which is defined as the rate at which the ejecta Lorentz factor decreases as a function of radius: $\Gamma \propto R^{-g} \propto t^{-g /(1+2 g)}$. In the Model 1 case, the best fit to the high-frequency evolution from 2.48 to 12.46 day is obtained for $g \approx 3.5$. However, this model does not fit the lowfrequency data well for any value of $g$; it overpredicts the emission at 1.36 day and underpredicts the peak at 2.48 day. A perfect fit to the data below $19 \mathrm{GHz}$ at 1.36 and 2.48 day can be obtained for Model 2 with $g \approx 0.2$, but this model would strongly underpredict the emission at all frequencies at 6.8 day and beyond. Furthermore, theoretical constraints limit $g$ to the range $1.5 \leqslant g \leqslant 3.5$ for an ISM environment (Kobayashi \& Sari 2000); a value of $g<1.5$ would imply that the ejecta has outpaced the FS. The best overall fit for Model 2 is obtained for $g \approx 1.5$, which fits the SED at 1.36 day and the low-frequency
Table 3

Model Parameters

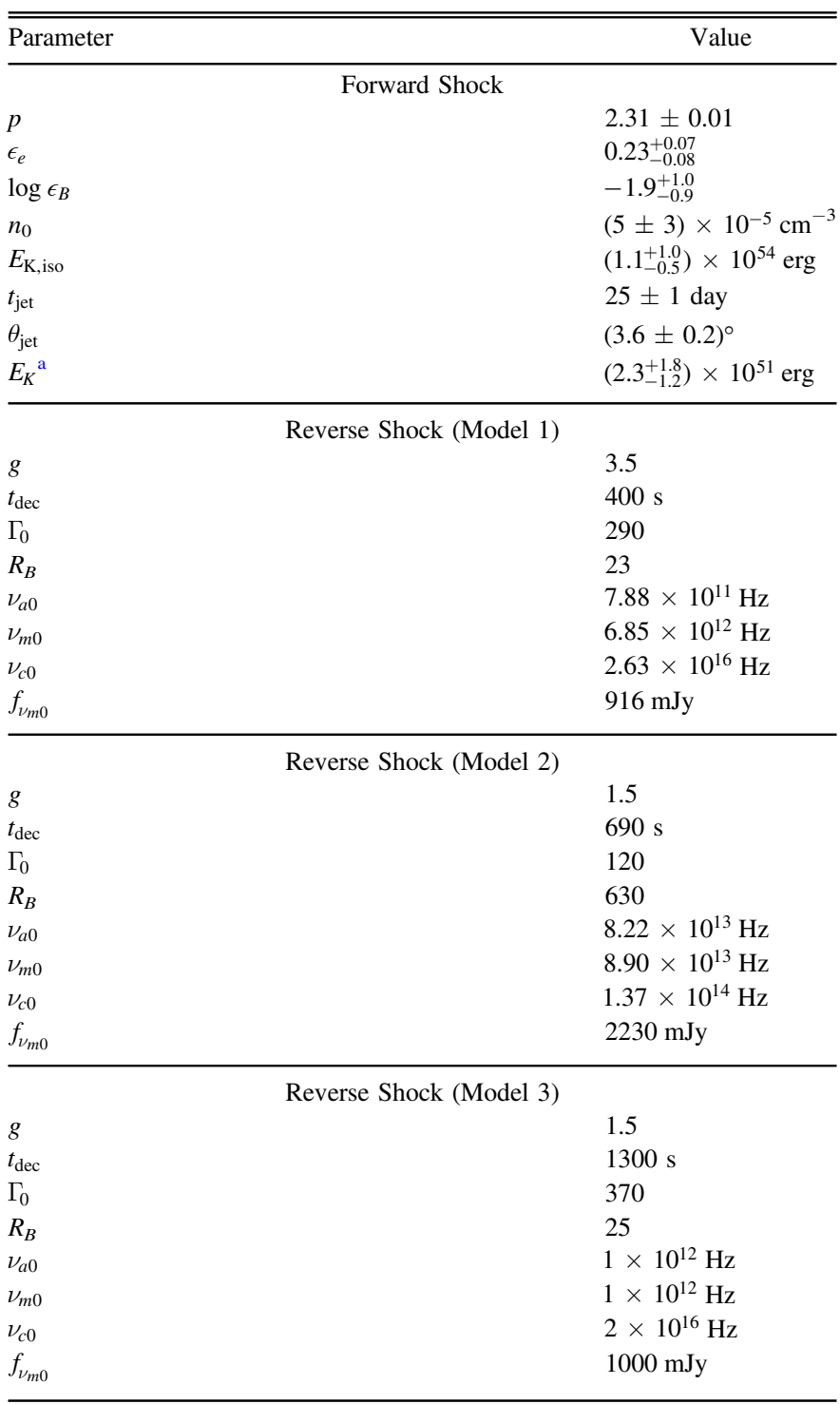

Note. The values given for each RS model are those plotted in Figures 1, 6, 7, and 8 , but a range of values are possible for each model (Section 5.1).

${ }^{a}$ Corrected for beaming.

observations at 6.8 day quite well but underpredicts the highfrequency flux density at 6.8 day. We show the best fits for Model 1 (red) and Model 2 (blue) together with the observed radio SEDs in Figure 6. Neither model reproduces the lowfrequency peak in epochs 4 and 5; we return to this point in Section 5.2. The ratio between the observed flux density and the model flux density at each frequency as a function of time is shown in Figure 7. Overall, Model 1 provides a better fit to the data at late times and higher frequencies, where we expect the flux distortions due to propagation effects to be smaller (shaded bands in Figures 6 and 7; Section 5.2).

A similar analysis can be carried out for relativistic RS models. These models are mainly distinguishable from the Newtonian RS models in their predictions for the early optical emission. Relativistic models where the peak frequency is defined by $\nu_{c} \approx 6 \mathrm{GHz}$ at 2.5 days are ruled out because they overpredict the observed optical emission $\approx 200-300 \mathrm{~s}$ after the 

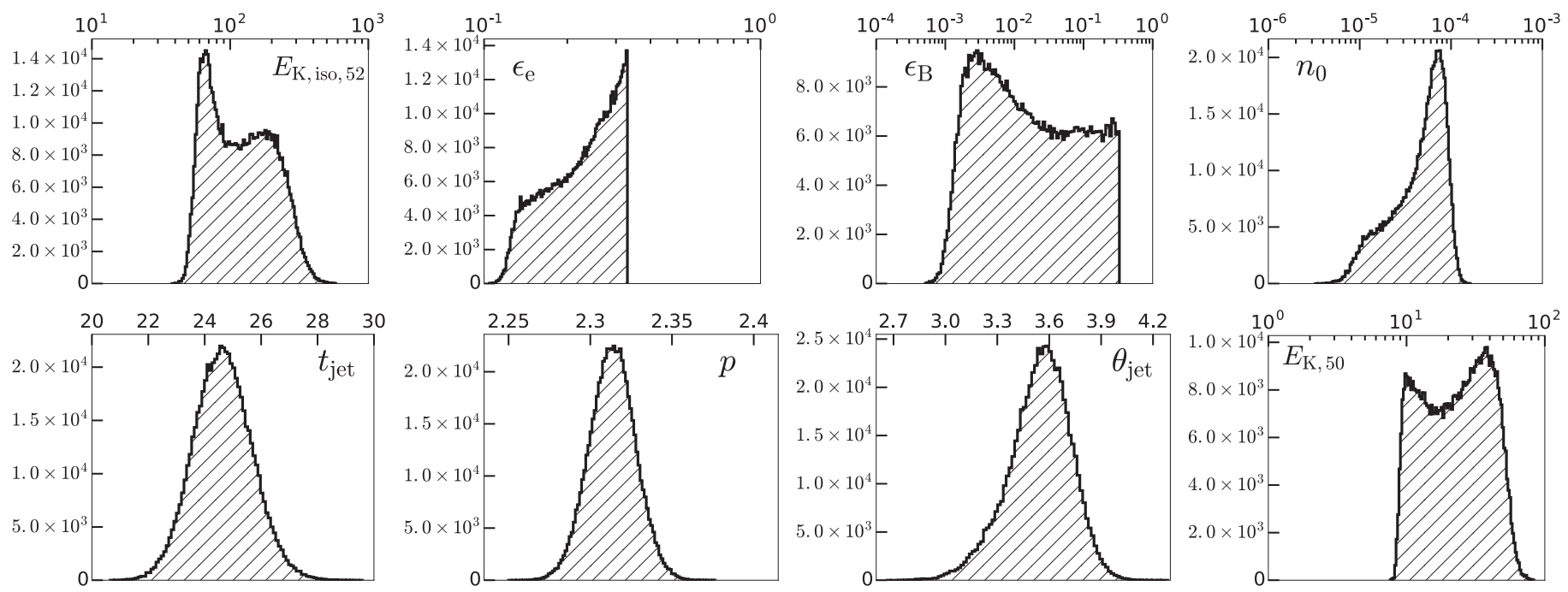

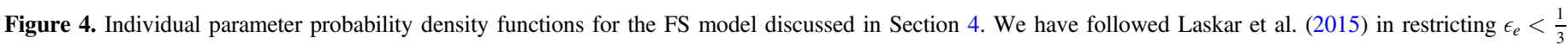
and $\epsilon_{B}<\frac{1}{3}$.

burst. Models where $\nu_{m, \mathrm{RS}} \lesssim \nu_{a, \mathrm{RS}} \approx 6 \mathrm{GHz}$ predict fluxes much lower than the observed optical fluxes at $t<0.03$ day, again implying that the optical emission originates separately (Model 3; Figure 8). Model 3 and Model 1 produce nearly identical radio SEDs at the times of our observations, so Model 3 is not shown in Figure 7. The exact parameter values chosen for plotting purposes are shown in Table 3 for each of the three RS models.

Consistency arguments require that the break frequencies of the RS and the FS are related at $t_{\mathrm{dec}}$, the time at which the RS finishes crossing the ejecta. This allows for a measurement of the bulk Lorentz factor $\left(\Gamma_{0}\right)$ and the RS magnetization $\left(R_{B} \equiv \epsilon_{B, \mathrm{RS}} / \epsilon_{B, \mathrm{FS}}\right)$ at this time (Gomboc et al. 2008; Harrison \& Kobayashi 2013). The loose constraints on one or more break frequencies in each model mean that we can only place limits on these quantities, rather than estimate them precisely. In particular, models with shorter $t_{\mathrm{dec}}$ values require larger values of $R_{B}$. For Models 1 and 3, we find $\Gamma_{0} \gtrsim 100$ and $1 \lesssim R_{B} \lesssim 100$, where $\Gamma_{0}$ is globally minimized for $R_{B} \approx 1$. The relativistic models require slightly longer deceleration times; $t_{\mathrm{dec}} \gtrsim 120 \mathrm{~s}$ for Model 1 , while $t_{\mathrm{dec}} \gtrsim 480 \mathrm{~s}$ for Model 3. For Model 2, we find $t_{\mathrm{dec}} \gtrsim 690 \mathrm{~s}$ and $R_{B} \gtrsim 630$. Model 2 cannot place any limits on $\Gamma_{0}$ because $\nu_{\mathrm{m}, \mathrm{RS}}$ is completely unconstrained in this case.

We can rule out some of these models by requiring $\epsilon_{B, \mathrm{RS}}<1 / 3$ as we did with $\epsilon_{B, \mathrm{FS}}$ in Section 4. From the distribution in Figure 4, we find that $\epsilon_{B, \mathrm{FS}}>1.56 \times 10^{-3}$ with 95\% confidence. This requires $R_{B}<214$, which is in tension with the lower limit on $R_{B}$ found for Model 2. For $\epsilon_{B, \mathrm{FS}}=0.0136$ (the median of the distribution), we require $R_{B}<25$ and the corresponding lower limit on $t_{\mathrm{dec}}$ increases, becoming $t_{\mathrm{dec}} \gtrsim 400 \mathrm{~s}$ for Model 1 and $t_{\mathrm{dec}} \gtrsim 1300 \mathrm{~s}$ for Model 3. The Model 3 limit is problematic because for relativistic RS models we expect $t_{\mathrm{dec}} \approx T_{90}$ (Kobayashi 2000). In GRB 160625B, weak $\gamma$-ray emission was observed until $\sim 10$ minutes after the LAT trigger time (Section 2.1), but even if we take $T_{90} \approx 600 \mathrm{~s}$ we find that $t_{\mathrm{dec}}$ is longer than expected unless $R_{B} \gtrsim 80$. We therefore conclude that Model 3 is consistent with the data but prefers lower values of $\epsilon_{B, \mathrm{FS}}$ than we would predict from the FS modeling alone. If this model is correct, it illustrates how additional information from the RS can break some of the FS parameter degeneracies we found in Section 4. A full FS + RS joint MCMC analysis is beyond the scope of this paper and would require better time sampling of the scattering effects discussed in the next section, which currently dominate the RS modeling uncertainties.

In summary, physical considerations clearly favor Model 1 or 3 over Model 2. Although we cannot distinguish between a relativistic and a Newtonian RS, both models place similar limits on the initial Lorentz factor and the magnetization of the ejecta, $\Gamma_{0} \gtrsim 100$ and $1 \lesssim R_{B} \lesssim 100$. Both models require a deceleration time longer than $T_{90}$ for the main $\gamma$-ray emission episode, slightly disfavoring Model 3 because relativistic RS models predict $t_{\mathrm{dec}} \approx T_{90}$. In future events, a joint analysis of well-sampled RS and FS components may enable better constraints on the burst parameters than is possible from observations of either component alone.

\subsection{Late-time Low-frequency Rebrightening: An Extreme Scattering Event (ESE)?}

The late-time radio emission from 12 to 22 days is characterized by an abrupt rebrightening centered at $3 \mathrm{GHz}$ that cannot be explained by the fading RS discussed above. Unlike the RS and FS synchrotron emission components, this component is spectrally narrow and only dominates the emission between 1-5 GHz. Furthermore, the peak flux density $F_{\nu, p}$ and peak frequency $\nu_{p}$ show unusual time evolution. We parameterize the time evolution of these quantities as $F_{\nu, p} \propto t^{a}$ and $\nu_{p} \propto t^{b}$, but find that the data are inconsistent with single values of $a$ and $b$. Between 12 and 22 days, $F_{\nu, p} \approx 0.5 \mathrm{mJy}$ and $\nu_{p} \approx 3 \mathrm{GHz}$ remain approximately constant. Before 12 days, the RS dominates the emission so the evolution of these quantities is poorly constrained, but we see that to hide the emission from this component at 7 days either $a$ or $b$ must be nonzero: we require $F_{\nu, p} \lesssim 0.1 \mathrm{mJy}$ or $\nu_{p} \gtrsim 25 \mathrm{GHz}$, implying $a \gtrsim 3$ or $b \lesssim-4$ from $7-12$ days. The excess vanishes by 48.34 day, implying $F_{\nu, p} \lesssim 0.1 \mathrm{mJy}$ or $\nu_{p} \lesssim 1.5 \mathrm{GHz}$ at this time and requiring $a \lesssim-2$ or $b \lesssim-0.5$ from $22-48$ days. Below, we present several possible explanations for this latetime component, considering both processes intrinsic to the burst and propagation effects that distort the radio spectrum. 

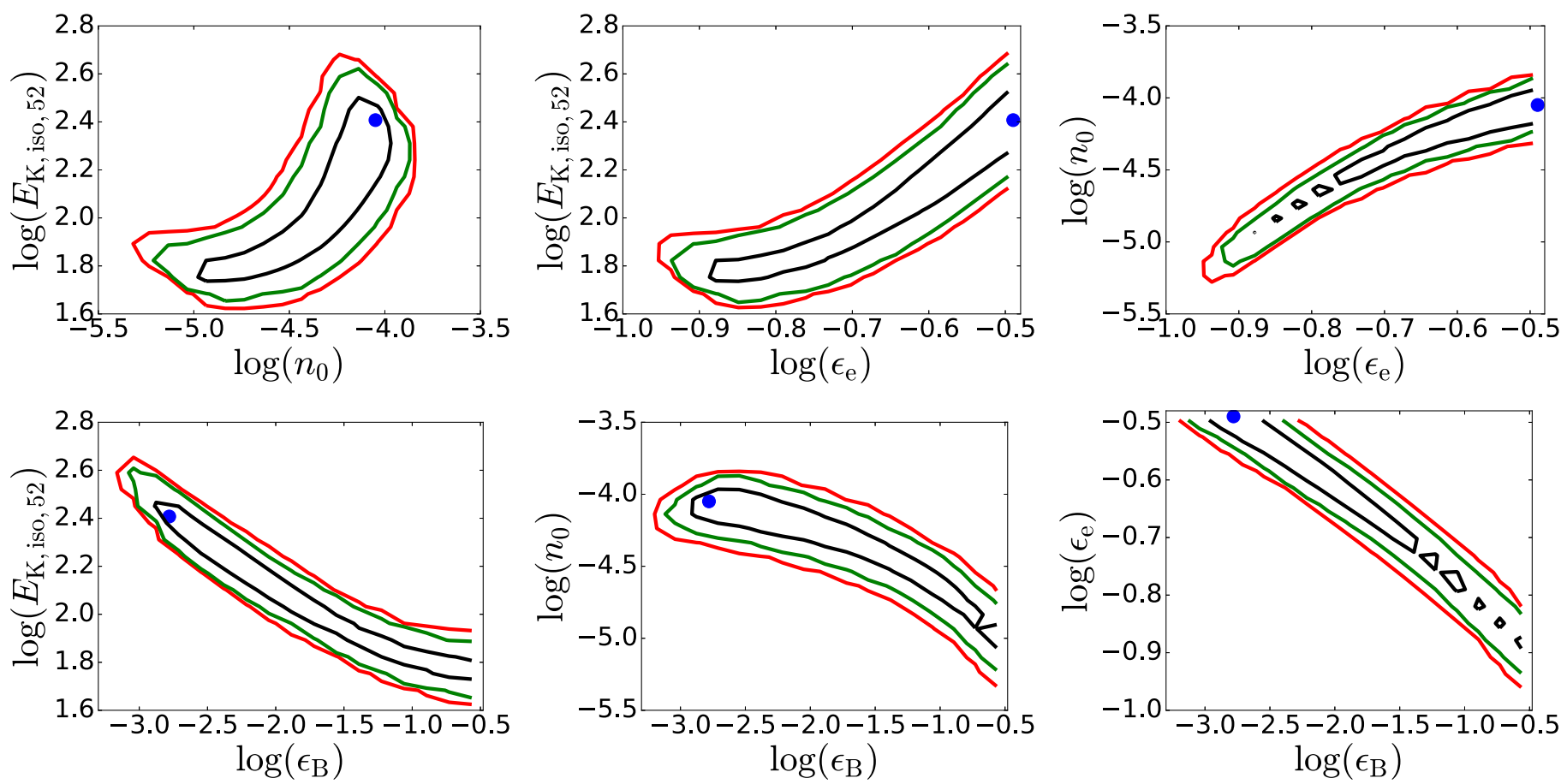

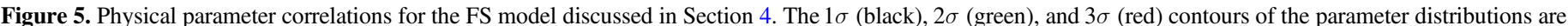

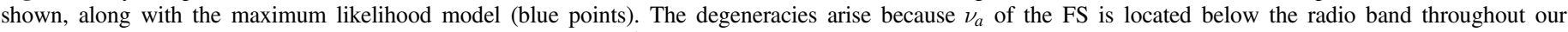
observations and is therefore only bounded at the upper end, $\nu_{a} \lesssim 1 \mathrm{GHz}$.

\subsubsection{Intrinsic Effects}

We first consider whether an additional synchrotron emission component, such as a second RS, can explain the late-time rebrightening. Like the FS and RS emission discussed above, its SED would consist of smooth power-law segments characterized by several break frequencies and an overall normalization. These break frequencies are predicted to evolve in time at constant rates $t^{b}$, but this is inconsistent with the variable time evolution described above, especially the rapid appearance of this emission component between 7 and 12 days. Furthermore, the narrowness of the emission component leads to spectral indices below and above the peak that are too sharp for standard RS or FS emission (Section 3.3).

Some of the problematic time evolution can be avoided if we consider a "refreshed" RS launched significantly after the prompt emission by the collision of two decelerated shells of ejecta with different initial Lorentz factors (Vlasis et al. 2011). The lack of radio emission from this component at $t<12$ days is expected if such a collision happens $\sim 10$ days after the GRB, but in such a model we would expect the peak flux and frequency of this component to decrease rapidly at $t>10$ day, inconsistent with the roughly constant flux we observe from 12-22 day. Furthermore, the collision would inject additional energy into the FS, so we would expect to see a late-time plateau or rebrightening at higher frequencies dominated by FS emission. The well-sampled $i^{\prime}$ band, $r^{\prime}$ band, and X-ray light curves show no deviations from smooth power-law decline preceding or during the appearance of the late-time radio component (Figure 1), so such models are ruled out. We conclude that neither a standard RS nor a "refreshed" RS can explain this emission.

Variability inconsistent with standard synchrotron afterglow models has been seen in X-ray and optical light curves of long GRBs previously (see Zhang 2007 for a review). X-ray and optical plateaus, flares, and rebrightenings have been variously attributed to late-time central engine activity, continuous energy injection from ejecta with a range of initial Lorentz factors that collide too gently to produce RS emission, structured jets, variations in microphysical parameters, and deviations of the circumburst density profile from a smooth constant or wind-like profile (Panaitescu et al. 2006; Lazzati \& Perna 2007; Kong et al. 2010; Uhm \& Zhang 2014; Laskar et al. 2015; Geng \& Huang 2016). Much of this unusual behavior takes place minutes to hours after the burst, rather than tens of days. Furthermore, all of these mechanisms are predicted to produce detectable emission at all frequencies, not just in the radio band, and we see no evidence of a broadband rebrightening in the X-rays or optical on any timescales probed by our observations (Figure 1). We conclude that the radio variability we observe in GRB $160625 \mathrm{~B}$ has a different origin from previously observed X-ray and optical variability in GRB afterglows.

To summarize, the late onset, long duration, and highly chromatic nature of the rebrightening are difficult to reconcile with any model in which this component is emission intrinsic to the source. We therefore consider models in which the emitted SED is distorted by propagation effects between the point of emission and the observer.

\subsubsection{Interstellar Scintillation}

Inhomogeneities in the electron density distribution along the line of sight cause ISS, which distorts radio waves propagating through the Galactic ISM and produces observable flux variations in compact extragalactic radio sources like GRB afterglows and quasars (Rickett 1990; Goodman 1997; Walker 1998; Goodman \& Narayan 2006). ISS is strongly frequency dependent: at high radio frequencies only modest flux variations are expected, while at low frequencies both strong diffractive and refractive effects are important. In the standard 



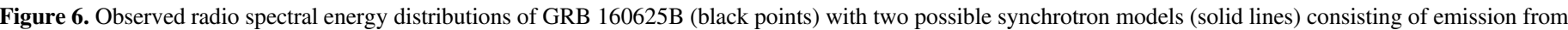

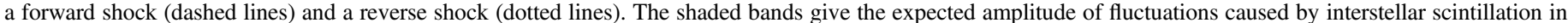

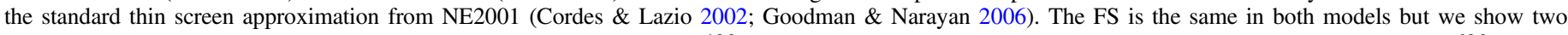



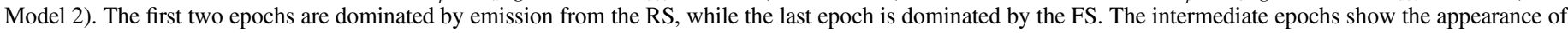

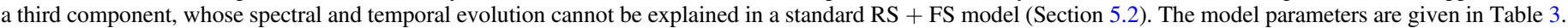
Model 2 provides a better fit to epoch 1, but a worse fit to the high-frequency data in epochs $2-4$.

picture, all scattering is assumed to occur at a single "thin screen" located at a distance determined by the NE2001 model for the Galactic electron distribution (Cordes \& Lazio 2002), typically $\sim 1 \mathrm{kpc}$ for high Galactic latitudes. We use this assumption to estimate the transition frequency between strong and weak scattering, $\nu_{T} \sim 15 \mathrm{GHz}$ for GRB 160625B. In the strong ISS regime, diffractive scintillation can produce large flux variations on timescales of minutes to hours but is only coherent across a bandwidth $\Delta \nu / \nu=\left(\nu / \nu_{T}\right)^{3.4}$ (Goodman 1997; Walker 1998). Since the typical bandwidth of our radio observations is about $1 \mathrm{GHz}$, we only expect diffractive scintillation to contribute significantly to the observed variability near $\nu_{T}$. Refractive scintillation is broadband and varies more slowly, on timescales of hours to days. In all regimes, the expected strength of the modulation decreases with time at all frequencies as the size of the emitting region expands, with diffractive ISS quenching before refractive ISS. The source expansion also increases the typical timescale of the variations for both diffractive and refractive ISS.

The shaded bands in Figure 6 show the expected strength of ISS in each of our radio epochs based on this simple picture, following Goodman \& Narayan (2006) and including both diffractive and refractive contributions. Clearly, the standard approach cannot explain the full amplitude of the lowfrequency peak at 12 and 22 days, although some of the deviations from the RS models explored in Section 5.1 are likely explained by ISS. The large amplitude of this component in the context of ISS suggests diffractive rather than refractive ISS. The spectral width of this feature $\Delta \nu / \nu \sim 1$ and the fact that the variability abruptly cuts off above $3.5 \mathrm{GHz}$ together suggest that $\nu_{T} \sim 3.5 \mathrm{GHz}$ (rather than $15 \mathrm{GHz}$ as determined from the NE2001 model). The value of $\nu_{T}$ is given by $\nu_{T} \approx 11.6\left(d_{\mathrm{scr}} / 1 \mathrm{kpc}\right)^{5 / 17} \mathrm{GHz}$, implying that the scattering screen is located at a distance of $d_{\text {scr }} \approx 20$ pc (Goodman 1997). The timescale for diffractive ISS at $2.7 \mathrm{GHz}$ is $\approx 30$ minutes, much shorter than the $\approx 10$ days that the excess endures, but longer than the time on source in each epoch (14 minutes). We see no evidence of variability at $2.7 \mathrm{GHz}$ within a single observation, but the signal-to-noise ratio is low. With only two observations during this time period, it is possible that we caught an upward fluctuation twice.

Since diffractive ISS is only effective for compact sources, we can use the duration of the observed variability to obtain an independent estimate of the size of the emitting region. The maximum angular size for diffractive scintillation at $2.7 \mathrm{GHz}$ is $\theta_{s}=94(\nu / 10 \mathrm{GHz})^{6 / 5}\left(d_{\mathrm{scr}} / \mathrm{kpc}\right)^{-1} \approx 20 \mu$ as for a screen distance $d_{\text {scr }}=20 \mathrm{pc}$ (Goodman 1997). The strong variability is not present in our final epoch, so we assume that the angular size of the afterglow increased past $\theta_{s}$ sometime between 22 and 48 days. Our FS model predicts that the angular size of the afterglow is $40 \mu$ as at 22 day and $60 \mu$ as at 48 day, which is consistent with this limit to within a factor of two. Exactly matching the FS prediction would require a slightly closer screen at $\approx 7-10 \mathrm{pc}$, which is also roughly consistent with the low-frequency observations. In Figure 7, we show the predicted $1 \sigma$ variations due to ISS for $d_{\mathrm{scr}}=10 \mathrm{pc}$ (magenta shaded region) and the standard NE2001 prediction $d_{\text {scr }}=2.2 \mathrm{kpc}$ (gray shaded region). The $d_{\mathrm{scr}}=10 \mathrm{pc}$ model does a better job of explaining the variability at frequencies below $5 \mathrm{GHz}$, but underpredicts the observed variations at $7-11 \mathrm{GHz}$ in epoch 1 . 



Time (days)

Figure 7. Radio light curves of GRB 160625B constructed by dividing the observed flux density in each band by the FS model given in Table 3 plus one of two Newtonian RS models. The red points show RS Model 1 and the blue points show RS Model 2 (Table 3; Section 5.1). The shaded bands show the Goodman \& Narayan (2006) $1 \sigma$ amplitude of ISS fluctuations at each frequency as a function of time using the NE2001 model (gray; $d_{\mathrm{scr}}=2.2 \mathrm{kpc}$ ) and a model with $d_{\mathrm{scr}}=10 \mathrm{pc}$ (magenta). The bandwidth of the observations at each frequency is $\sim 1 \mathrm{GHz}$, except at 1.45 and $1.77 \mathrm{GHz}$, where it is $\sim 250 \mathrm{MHz}$. The observed variability appears correlated over bandwidths of a few $\mathrm{GHz}$ and has an amplitude and duration similar to chromatic "cusps" previously attributed to plasma lensing of quasars (Fiedler et al. 1987, 1994; Bannister et al. 2016).

Both models fail to reproduce the late-time flux deficit at high frequencies noted in Section 3.1, although many of these points have large error bars due to the faintness of the fading afterglow.

GRB 160625B is not the first source in which non-standard ISS models have been invoked to explain extreme variability. An even closer scattering screen $\left(d_{\text {scr }}=1-2\right.$ pc) was previously inferred for the quasar $\mathrm{J} 1819+3845$, which showed extreme variability that stopped abruptly after 7.5 years and did not return in a further 6 years of monitoring (de Bruyn \& Macquart 2015). The limited duration of the J1819+3845 variability suggests that the scattering screen was compact or patchy, which may also be the case for the nearby structure responsible for the strong flux modulations we see in GRB 160625B. We note that the extreme amplitude, bandwidth, and duration of this component are also qualitatively similar to ESEs observed in quasars (Fiedler et al. 1987, 1994; Bannister et al. 2016). While ISS has been observed in other GRB afterglows (e.g., Waxman et al. 1998; Berger et al. 2003; Chandra et al. 2008), this would make GRB 160625B the first GRB to exhibit an ESE. The proposed cause of ESEs is lensing by dense au-scale plasma structures in the Milky Way that transit the line of sight. Such structures are not dissimilar to the $\sim 100$ au-scale object proposed as the cause of the extreme variability in J1819+3845 (de Bruyn \& Macquart 2015). As with the Fiedler et al. (1994) ESEs, the variability in GRB $160625 \mathrm{~B}$ is uncorrelated across bandwidths larger than a few $\mathrm{GHz}$ (Figure 7). In other literature ESEs, a rapid flux enhancement is followed by an extended period ( $\sim$ months) in which the flux is suppressed and then by a second enhancement, producing chromatic symmetric U-shaped features. The amplitude $(\approx 3$ times the predicted model flux) and duration $(\approx 10$ days $)$ of the $2.7 \mathrm{GHz}$ feature are comparable to the flux enhancements seen during these bracketing cusps. A search for long-lasting flux suppression before or after the observed enhancement is complicated by uncertainties in the afterglow modeling, limited wavelength coverage before 6 days increased flux uncertainties at later times due to the fading of the afterglow, and the more sparse time sampling after 12 days. We note that the rapid flux variations at $1-6$ day at $5-9 \mathrm{GHz}$ are somewhat reminiscent of the sharp features observed at 8.5 GHz in an ESE toward the quasar $0954+658$ during the $2.7 \mathrm{GHz}$ event minimum (Fiedler et al. 1987), which would mean that the observed flux increase in GRB 160625B corresponds to the end of the proposed ESE.

We conclude that the excess low-frequency emission observed in GRB 160625B from 12-22 day is broadly consistent with previously observed variability in compact 

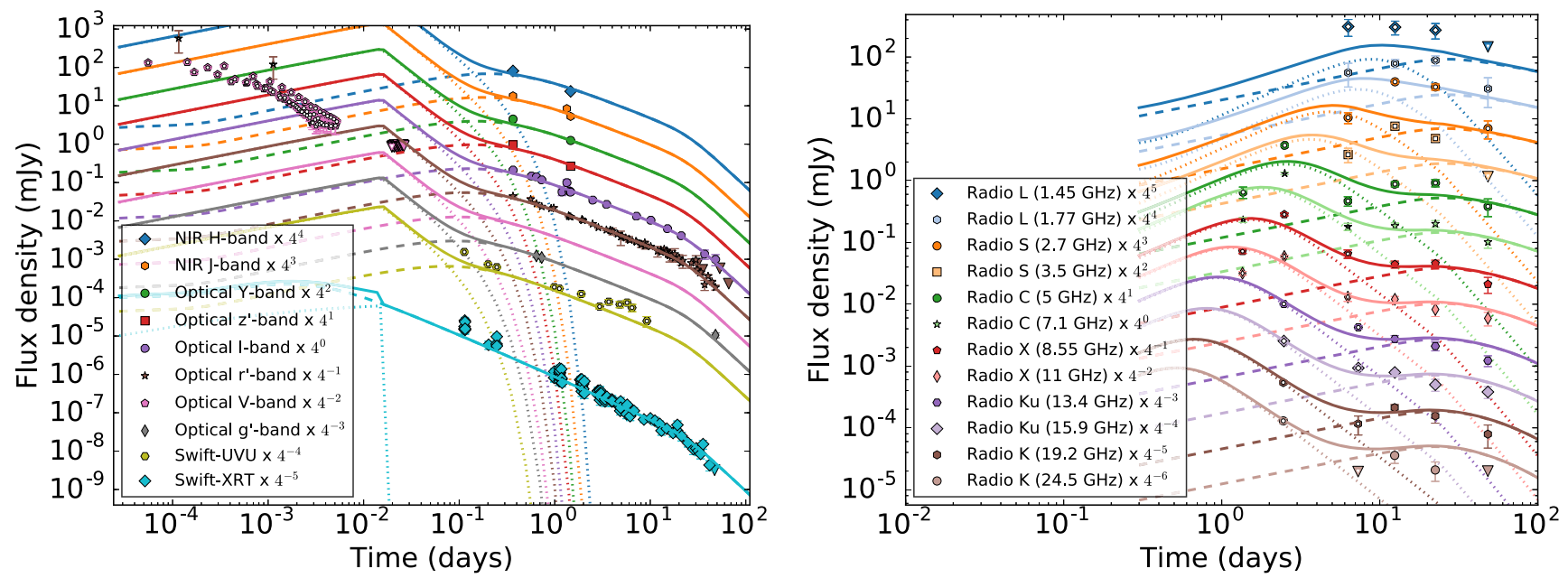

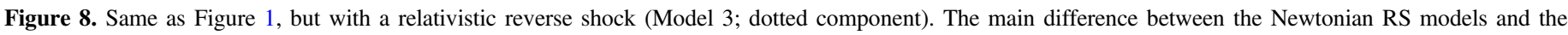
relativistic model shown here is the early optical behavior (left). The fit to the radio data (right) is comparable to Model 1 (Figure 1; Section 5.1).

extragalactic sources attributed to diffractive ISS or other extreme scattering effects. The observations suggest that much of the scattering occurs at a distance of $\approx 10-20 \mathrm{pc}$, much closer than is typically assumed. A combination of scattering from this nearby screen and the more distant "standard" screen could explain the additional variability observed at 1.4-6.8 day. Future GRB observations with broad frequency coverage and denser time sampling will better constrain the timescales of such variability and allow us to disentangle ISS from variations intrinsic to the source.

\section{Conclusions}

We presented detailed observations of the long-duration GRB 160625B spanning radio to X-ray wavelengths and found that the data are mostly well fit by the standard FS model for GRB afterglows. We use a MCMC analysis to constrain the afterglow properties and find that GRB 160625B is a highly energetic event that exploded in an ISM-like low-density medium. Our early multi-frequency radio observations show a clear excess compared to the standard predictions for synchrotron emission from a FS. We interpret this excess as a RS, making GRB 160625B only the third GRB for which an in-depth study of RS emission at multiple epochs has been possible. All three events occurred in low-density environments, suggesting that such conditions are particularly favorable for the production of strong, long-lasting RS emission. Our ability to constrain the jet properties is restricted by the limited wavelength coverage of our first epoch and by the additional uncertainty introduced by ISS, which causes large random flux perturbations at low frequencies in our first five radio epochs. We place a lower limit on the initial bulk Lorentz factor of the ejecta of $\Gamma_{0} \gtrsim 100$ that is robust to other uncertainties in the RS modeling, confirming the highly relativistic nature of the outflow. The magnetization of the $\mathrm{RS}$ is $R_{B} \approx 1-100$.

One key finding from this analysis is that propagation effects cannot be ignored when attempting detailed physical characterization of GRB radio afterglows, especially at early times when RS emission is most relevant. The radio afterglow of GRB 160625B shows unusual variability on a range of timescales, most notably a low-frequency rebrightening centered at $3 \mathrm{GHz}$ at $12-22$ days. This late-time excess cannot be easily explained with processes intrinsic to the source. Instead, it is more naturally explained in the context of propagation effects in the Galactic ISM, and is roughly consistent with strong diffractive scintillation by a thin screen with an effective distance of $\approx 10-20 \mathrm{pc}$. The extreme variability at $2.7 \mathrm{GHz}$ is qualitatively similar to plasma lensing by compact structures in the Milky Way. A more detailed analysis of this intriguing similarity is not possible for GRB 160625B because our observing strategy, while a significant improvement on previous efforts, is optimized to probe RS emission at early times rather than more rapid ISS-induced variability that may endure for several weeks. Disentangling propagation and intrinsic effects will require denser time and frequency coverage of GRB radio afterglows than has been attempted to date, but will enable new probes of both GRB physics and the nature of turbulent structures in the ISM. We will further explore the impact of propagation effects on GRB afterglows in future work.

We thank R. Barniol Duran, M. Johnson, R. Narayan, R. Sari, D. Warren, B.-B. Zhang, and the attendees of the Eighth Huntsville Gamma-Ray Burst Symposium for useful conversations. We also thank the anonymous referee for helpful comments that have improved this manuscript. K.D.A. and E.B. acknowledge support from NSF grant AST-1411763 and NASA ADA grant NNX15AE50G. T.L. is a Jansky Fellow of the National Radio Astronomy Observatory (NRAO). W.F. is supported by NASA through Einstein Postdoctoral Fellowship grant number PF4-150121. VLA observations were taken as part of our VLA Large Program 15A-235 (PI: E. Berger). The VLA is operated by the NRAO, a facility of the National Science Foundation operated under cooperative agreement by Associated Universities, Inc. This work made use of data supplied by the UK Swift Science Data Centre at the University of Leicester.

Software: CASA (McMullin et al. 2007), pwkit (Williams et al. 2017).

\section{ORCID iDs}

K. D. Alexander (ib https://orcid.org/0000-0002-8297-2473

E. Berger (i) https://orcid.org/0000-0002-9392-9681

W. Fong (i) https://orcid.org/0000-0002-7374-935X

S. Kobayashi (iD https://orcid.org/0000-0001-7946-4200 
N. R. Tanvir (iD https://orcid.org/0000-0003-3274-6336

P. K. G. Williams (iD https://orcid.org/0000-0003-3734-3587

\section{References}

Atwood, W. B., Abdo, A. A., Ackermann, M., et al. 2009, ApJ, 697, 1071 Bannister, K. W., Stevens, J., Tuntsov, A. V., et al. 2016, Sci, 351, 354 Batsch, T., Castro-Tirado, A. J., Czyrkowski, H., et al. 2016, GCN, 19615, 1 Beniamini, P., \& van der Horst, A. J. 2017, arXiv:1706.07817

Berger, E., Kulkarni, S. R., Pooley, G., et al. 2003, Natur, 426, 154 Brown, T. M., Baliber, N., Bianco, F. B., et al. 2013, PASP, 125, 1031 Burns, E. 2016, GCN, 19581, 1

Chambers, K. C., Magnier, E. A., Metcalfe, N., et al. 2016, arXiv:1612.05560 Chandra, P., Cenko, S. B., Frail, D. A., et al. 2008, ApJ, 683, 924

Chevalier, R. A., \& Li, Z.-Y. 2000, ApJ, 536, 195

Cordes, J. M., \& Lazio, T. J. W. 2002, arXiv:astro-ph/0207156

de Bruyn, A. G., \& Macquart, J.-P. 2015, A\&A, 574, A125

Dirirsa, F., Racusin, J., McEnery, J., \& Desiante, R. 2016, GCN, 19580, 1

Evans, P. A., Beardmore, A. P., Page, K. L., et al. 2007, A\&A, 469, 379

Evans, P. A., Beardmore, A. P., Page, K. L., et al. 2009, MNRAS, 397, 1177

Fiedler, R., Dennison, B., Johnston, K. J., Waltman, E. B., \& Simon, R. S 1994, ApJ, 430, 581

Fiedler, R. L., Dennison, B., Johnston, K. J., \& Hewish, A. 1987, Natur, 326, 675

Foreman-Mackey, D., Hogg, D. W., Lang, D., \& Goodman, J. 2013, PASP, 125,306

Gehrels, N., Chincarini, G., Giommi, P., et al. 2004, ApJ, 611, 1005

Geng, J. J., \& Huang, Y. F. 2016, AdAst, 2016, 159214

Gomboc, A., Kobayashi, S., Guidorzi, C., et al. 2008, ApJ, 687, 443

Goodman, J. 1997, NewA, 2, 449

Goodman, J., \& Narayan, R. 2006, ApJ, 636, 510

Granot, J., \& Sari, R. 2002, ApJ, 568, 820

Harrison, R., \& Kobayashi, S. 2013, ApJ, 772, 101

Japelj, J., Kopač, D., Kobayashi, S., et al. 2014, ApJ, 785, 84

Karpov, S., Beskin, G., Bondar, S., et al. 2016, GCN, 19603, 1

Kobayashi, S. 2000, ApJ, 545, 807

Kobayashi, S., \& Sari, R. 2000, ApJ, 542, 819

Kong, S. W., Wong, A. Y. L., Huang, Y. F., \& Cheng, K. S. 2010, MNRAS, 402, 409

Kopač, D., Kobayashi, S., Gomboc, A., et al. 2013, ApJ, 772, 73

Kopač, D., Mundell, C. G., Kobayashi, S., et al. 2015, ApJ, 806, 179

Kuroda, D., Hanayama, H., Miyaji, T., et al. 2016, GCN, 19599, 1

Laskar, T., Alexander, K. D., Berger, E., et al. 2016, ApJ, 833, 88

Laskar, T., Berger, E., Margutti, R., et al. 2015, ApJ, 814, 1
Laskar, T., Berger, E., Tanvir, N., et al. 2014, ApJ, 781, 1

Laskar, T., Berger, E., Zauderer, B. A., et al. 2013, ApJ, 776, 119

Lazzati, D., \& Perna, R. 2007, MNRAS, 375, L46

Lü, H.-J., Lü, J., Zhong, S.-Q., et al. 2017, arXiv:1702.01382

McMullin, J. P., Waters, B., Schiebel, D., Young, W., \& Golap, K. 2007, in ASP Conf. Ser. 376, Astronomical Data Analysis Software and Systems XVI, ed. R. A. Shaw, F. Hill, \& D. J. Bell (San Francisco, CA: ASP), 127 Meegan, C., Lichti, G., Bhat, P. N., et al. 2009, ApJ, 702, 791

Melandri, A., D’Avanzo, P., D'Elia, V., et al. 2016, GCN, 19585, 1

Melandri, A., Kobayashi, S., Mundell, C. G., et al. 2010, ApJ, 723, 1331

Meszaros, P., \& Rees, M. J. 1993, ApJ, 405, 278

Mundell, C. G., Melandri, A., Guidorzi, C., et al. 2007, ApJ, 660, 489

Oates, S. 2016, GRB Coordinates Network, Circular Service, 19589, 1

Oke, J. B., \& Gunn, J. E. 1983, ApJ, 266, 713

Panaitescu, A., Mészáros, P., Gehrels, N., Burrows, D., \& Nousek, J. 2006, MNRAS, 366, 1357

Perley, D. A., Cenko, S. B., Corsi, A., et al. 2014, ApJ, 781, 37

Rickett, B. J. 1990, ARA\&A, 28, 561

Roming, P. W. A., Kennedy, T. E., Mason, K. O., et al. 2005, SSRv, 120 95

Sari, R., \& Esin, A. A. 2001, ApJ, 548, 787

Sari, R., \& Piran, T. 1999, ApJ, 520, 641

Sari, R., Piran, T., \& Halpern, J. P. 1999, ApJL, 519, L17

Sari, R., Piran, T., \& Narayan, R. 1998, ApJL, 497, L17

Schlafly, E. F., \& Finkbeiner, D. P. 2011, ApJ, 737, 103

Steele, I. A., Smith, R. J., Rees, P. C., et al. 2004, Proc. SPIE, 5489, 679

Uhm, Z. L., \& Zhang, B. 2014, ApJ, 789, 39

Vlasis, A., van Eerten, H. J., Meliani, Z., \& Keppens, R. 2011, MNRAS, 415,279

Walker, M. A. 1998, MNRAS, 294, 307

Wang, Y.-Z., Wang, H., Zhang, S., et al. 2017, ApJ, 836, 81

Watson, A. M., Troja, E., Butler, N., et al. 2016, GCN, 19602, 1

Waxman, E., Kulkarni, S. R., \& Frail, D. A. 1998, ApJ, 497, 288

Williams, P. K. G., Clavel, M., Newton, E., \& Ryzhkov, D. 2017, Pwkit: Astronomical utilities in Python, Astrophysics Source Code Library, ascl:1704.001

Willingale, R., Starling, R. L. C., Beardmore, A. P., Tanvir, N. R., \& O'Brien, P. T. 2013, MNRAS, 431, 394

Woosley, S. E., \& Bloom, J. S. 2006, ARA\&A, 44, 507

Xu, D., Malesani, D., Fynbo, J. P. U., et al. 2016, GCN, 19600, 1

Zacharias, N., Finch, C., Subasavage, J., et al. 2015, AJ, 150, 101

Zhang, B. 2007, ChJAA, 7, 1

Zhang, B., Liang, E., Page, K. L., et al. 2007, ApJ, 655, 989

Zhang, B.-B., Zhang, B., Castro-Tirado, A. J., et al. 2016, arXiv:1612.03089 